\title{
Nicotinamide Supplementation Attenuates Renal Interstitial Fibrosis via Boosting the Activity of Sirtuins
}

\author{
Xin Zhen Shaowu Zhang Feifei Xie Miaomiao Zhou Zheng Hu \\ Fengxin Zhu Jing Nie
}

State Key Laboratory of Organ Failure Research, National Clinical Research Center of Kidney Disease, Key Laboratory of Organ Failure Research (Ministry of Education), Division of Nephrology, Nanfang Hospital, Southern Medical University, Guangzhou, China

\section{Keywords}

Nicotinamide · Renal interstitial fibrosis · Sirtuins · G2/M arrest $\cdot$ Inflammation

\begin{abstract}
Background: Progressive tubulointerstitial fibrosis (TIF) is the final common pathway leading to ESRD. There is an urgent need to develop effective approaches slowing the progression of TIF. Previous studies showed that systemic supplementation of nicotinamide (NAM) increases renal NAD ${ }^{+}$ and reverses ischemic-reperfusion induced acute renal injury. However, the role and mechanism of NAM in TIF has been unclear. Methods: In vivo, we injected NAM $(0.25 \mathrm{mg} / \mathrm{g})$ 3 days before unilateral ureter obstruction (UUO) till day 7 post-operation. In vitro, mouse primary proximal tubular epithelial cells (PTCs), rat renal NRK-49F cells, and human renal proximal tubular epithelial cell (HK-2) were pretreated with the indicated concentration of NAM $1 \mathrm{~h}$ before incubation with transform growth factor- $\beta 1$ (TGF- $\beta 1$ ) or aristolochic acid (AA) for 24 or $48 \mathrm{~h}$. To evaluate the role of sirtuins (SIRTs), PTCs were pretreated with EX527 or resveratrol 30 min before incubation with NAM and TGF- $\beta 1$. Results: In the present study, we demonstrated that NAM supplementation prevented UUO-induced TIF, and AA-induced renal injury.
\end{abstract}

(C) 2021 The Author(s)

Published by S. Karger AG, Basel

This article is licensed under the Creative Commons AttributionNonCommercial-NoDerivatives 4.0 International License (CC BY NC-ND) (http://www.karger.com/Services/OpenAccessLicense). Usage and distribution for commercial purposes as well as any distribution of modified material requires written permission.
NAM also decreased the expression of pro-fibrotic proteins and pro-inflammatory cytokines (IL- 6 and TNF-a) and attenuated interstitial inflammation. In vitro experiment showed that, NAM inhibited AA-induced G2/M arrest of HK-2 cells by downregulating the expression of cyclin G1, a target gene of p53. In addition, NAM inhibited TGF- $\beta 1$-induced fibroblast proliferation and activation shown as downregulated expression of collagen I, fibronectin, PCNA, cyclin D1, IL-6, and TNF-a. NAM decreased the acetylation of Smad3 and p53. EX527, an inhibitor of SIRT1, reversed the effect of NAM on TGF- $\beta 1$-induced matrix protein production. However, resveratrol, a SIRT1 activator, did not further boost the protective effect of NAM on reducing matrix protein production. Conclusions: Taken together, these data indicate that NAM supplementation could inhibit TIF at least partially by boosting the activity of sirtuins.

(c) 2021 The Author(s)

Published by S. Karger AG, Basel

\section{Introduction}

Progressive tubulointerstitial fibrosis (TIF), which is characterized by myofibroblast activation and excessive deposition of extracellular matrix (ECM) [1], is the final common pathway leading to ESRD, irrespective of the 
initial cause [2]. Unfortunately, there are no approved medicines for the treatment of TIF [3]. Novel anti-fibrotic therapeutic approaches are urgently needed to reduce the burden of CKD.

In the progression of TIF, tubular cell injury remains a central event [4], and its contribution to the progression of fibrogenesis has been extensively studied. A strong correlation has been reported between G2/M arrest of proximal tubular cells and the outcome of TIF $[5,6]$. The prolonged G2/M arrest of proximal tubular cells after acute kidney injury (AKI) leads to overproduction of transform growth factor- $\beta 1$ (TGF- $\beta 1$ ) and connective tissue growth factor [7]. Most recent study showed that tubular cells in the G2-M phase form the target of rapamycin (TOR)-autophagy spatial coupling compartments (TASCCs), which promote pro-fibrotic and inflammatory secretion similar to the senescence-associated secretary phenotype and contribute to the interstitial inflammation of TIF [8].

Nicotinamide (NAM) is an amide form of vitamin B3, which is endogenously found in low quantities in mammalian cells. When supplemented in therapeutic doses, $\mathrm{NAM}$ is rapidly converted to $\mathrm{NAD}^{+}$through a salvage pathway $[9,10]$. The effects of NAM are largely mediated by a cellular increase of $\mathrm{NAD}^{+}[11]$. NAM can either contribute to de novo $\mathrm{NAD}^{+}$biosynthesis through the intermediate nicotinamide mononucleotide (NMN) [12] or undergo methylation to N1-methylnicotinamide (MNAM) followed by irreversible oxidation to waste products [13]. The increased $\mathrm{NAD}^{+}$in cells results in an increase in the ratio of $\mathrm{NAD}^{+} / \mathrm{NADH}$, a key cellular redox state that plays an important role in redox signaling and bioenergetics [14]. In addition, $\mathrm{NAD}^{+}$is also a substrate for non-redox enzymes that consume $\mathrm{NAD}^{+}$such as sirtuins (SIRTs), poly-ADP ribose polymerases (PARPs), and CD38/CD157 [15]. A wealth of data suggest that SIRT1, a member of the sirtuins family, plays a protective role in acute and chronic kidney injury $[16,17]$.

Chronic deficiency of NAM affects several metabolically active organs. Therapeutic effects of NAM have been reported in diverse diseases ranging from diet-induced obesity to neuronal degeneration [18]. Recent studies reported that de novo $\mathrm{NAD}^{+}$biosynthesis becomes impaired during AKI, resulting in reduced renal $\mathrm{NAD}^{+}$content [12]. Systemic supplementation of NAM increases renal $\mathrm{NAD}^{+}$and reverses ischemic-reperfusion (IR)-induced AKI. Moreover, NAM supplementation reduces the risk of AKI among adults undergoing cardiac surgery $[10,19]$.

In the current study, we reported a beneficial role of NAM supplementation in TIF induced by unilateral ure- teral obstruction (UUO) and tubular injury induced by aristolochic acid (AA). In vitro experiments demonstrated that NAM conducted an anti-fibrotic effect via blocking G2/M arrest of human renal proximal tubular epithelial (HK-2) cells and fibroblast activation by boosting the activity of SIRT1.

\section{Materials and Methods}

\section{Animal Model}

Eight-week-old male C57BL/6J mice were randomly divided into 4 groups and subjected to either sham operation or UUO as described previously [20]. (1) control group, (2) control + NAM group, (3) UUO group, and (4) UUO + NAM group. NAM (0.25 $\mathrm{mg} / \mathrm{g} /$ day) was given 3 days before operation till day 7 post-operation through intraperitoneal injection. All mice were sacrificed at day 7 post-surgery, and kidney tissues were frozen.

Eight-week-old male C57BL/6J mice were subject to intraperitoneal injection with AA sodium salt ( $3.5 \mathrm{mg} / \mathrm{kg}$, Sigma-Aldrich) every other day for 10 days, and then the mice were sacrificed on day 15 . The mice were intraperitoneally injected with NAM $(0.5$ $\mathrm{mg} / \mathrm{g} /$ day) 1 day before giving AA and continuously until the day before sacrifice and kidney tissues were frozen. All animal experiments were approved by the Ethics Committee for Animal Experiments of the Southern Medical University.

\section{Cell Culture and Treatment}

Mouse primary proximal tubular epithelial cells (PTCs) were separated with percoll buffer as described previously [21]. PTCs and NRK-49F cells were cultured in DMEM or DMEM/F12 medium supplemented with $10 \%$ fetal bovine serum (Gibco/Life Technologies, New York, USA). To evaluate the effects of NAM on the TGF- $\beta 1$-associated fibrosis, PTCs and NRK-49F cells were pretreated with NAM $(1,5,10$, or $20 \mathrm{mM})$ for $1 \mathrm{~h}$ before stimulation with $10 \mathrm{ng} / \mathrm{mL}$ TGF- $\beta 1$ (R\&D Systems, Minneapolis, MN, USA) for $24 \mathrm{~h}$. NAM used in the experiments was dissolved in normal saline.

To evaluate the role of SIRT1 in NAM improves TGF- $\beta 1$ associated fibrosis, PTCs were preincubated with EX527 $(0.5 \mu \mathrm{M}$, Selleck, Houston, TX, USA) or resveratrol (50 mM, Selleck, Houston, TX, USA) for $30 \mathrm{~min}$ and then treated with NAM (20 mM) for $30 \mathrm{~min}$. Thereafter, PTCs were incubated with $10 \mathrm{ng} / \mathrm{mL}$ TGF- $\beta 1$ for $24 \mathrm{~h}$. HK- 2 cells were cultured with DMED medium that contained $10 \%$ fetal bovine serum. When reached approximately $70 \%$ confluence, the cells were pretreated with NAM (10-20 mM) for $1 \mathrm{~h}$ before adding aristolochic acid $(15 \mu \mathrm{g} / \mathrm{mL})$ for $48 \mathrm{~h}$.

\section{RNA Extraction and Quantitative Real-Time PCR}

Total RNA was isolated from mouse primary PTCs or kidney using TRIzol reagent [22], and cDNA was synthesized according to the manufacturer's instructions (Invitrogen, Carlsbad, CA, USA). Real-time PCR was performed on an ABI Step-1 Plus sequence detection system using SYBR Green Master Mix (Vazyme). The expression levels of mRNAs were calculated after normalizing with GAPDH by the comparative CT method $\left(2^{-\Delta \Delta \mathrm{Ct}}\right)$. The primer sequences used in the experiments were described as follows: 
NNMT (mice) forward 5'-TGTGCAGAAAACGAGATCCTC-3'; reverse 5'-AGTTCTCCTTTTACAGCACCCA-3'; GAPDH (mice) forward 5'-ACTCCACTACGGCAAATTC-3'; reverse 5'-TCTCCATGGTGGTGAAGACA-3'; NGAL (mice) forward 5'-GCAGGTGGTACGTTGTGGG-3'; reverse 5'-CTCTTGTAGCTCATAGATGGTGC-3'; NAMPT (mice) forward 5'-AATGTCTCCTTCGGTTCTGG-3'; reverse 5'-GCAACTGGGTCCTTAAACACA-3'; NMNAT1 (mice) forward 5'-TGGCTCTTTTAACCCCATCAC-3'; reverse 5'-TCTTCTTGTACGCATCACCGA-3'; NMNAT3 (mice) forward 5'-CCTGTGGTTCCTTCAACCCC-3'; reverse 5'-AGATGATGCCCTCAATCACCT-3'; IL-6 (mice) forward 5'-TAGTCCTTCCTACCCCAATTTCC-3'; reverse 5'-CTGTTGTTCAGACTCTCTCCCT-3'; TNF-a (mice) forward 5'-CAGGCGGTGCCTATGTCTC-3'; reverse 5'-CGATCACCCCGAAGTTCAGTAG-3'; MCP-1 (mice) forward 5'-TTAAAAACCTGGATCGGAACCAA-3'; reverse 5'-GCATTAGCTTCAGATTTACGGGT-3'; IL-1 $\beta$ (mice) forward 5'-GCAACTGTTCCTGAACTCAACT-3'; reverse 5'-ATCTTTTGGGGTCCGTCAACT-3'; CG1 (mice) forward 5'-ACAACTGACTCTCAGAAACTGC-3'; reverse 5'-CATTATCATGGGCCGACTCAAT-3'; IL-6 (rat) forward 5'-CTCTCCGCAAGAGACTTCCAG-3'; reverse 5'-TGTGGGTGGTATCCTCTGTGA-3'; MCP-1 (rat) forward 5'-CTCCACCACTATGCAGGTCTC-3'; reverse 5'-GGGCATTAACTGCATCTGGCT-3'; GAPDH (rat) forward 5'-ACCATCTTCCAGGAGCGAGA-3'; reverse 5'-CTCGTGGTTCACACCCATCA-3'.

\section{Western Blot Analysis}

Cells and kidney tissues were homogenized in RIPA lysis buffer (50 mM Tris, pH 7.4, $150 \mathrm{~mm} \mathrm{NaCl,1 \%}$ Triton X-100, 0.5\% sodium deoxycholate, and $0.1 \%$ SDS) with $1 \%$ protease inhibitor cocktail for $30 \mathrm{~min}$ on ice. Samples were electrophoresed and electrotransferred to acid membranes (Millipore, Bedford, MA, USA). The membranes were immunoblotted with indicated primary antibodies. Horseradish peroxidase-conjugated or fluorescent labeled anti-rabbit or anti-mouse antibodies (CST Signaling, Danvers, $\mathrm{MH}$, USA) were used as secondary antibodies [23]. The blots were visualized by using an enhanced chemiluminescence detection system (Perkin Elmer Life Science, Boston, MA, USA) or Odyssey scanning. The primary antibodies used in this experiment were listed as follows: anti-N-methyltransferase (NNMT) (ab119758) and anti-tenascin C (ab108930) were purchased from Abcam (Cambridge, UK). Anti-p-Smad3 (\#9520), anti-Smad3 (\#9523), anti-pSmad2 (\#18338), anti-Smad2 (\#5339), anti-p-STAT3 (\#9145), anti-STAT3 (\#12640), anti-forkhead box O1 (FoxO1) (\#2880), anti-cyclin D1 (\#2978), and anti-acetylated-lysine (\#6952, \#9441) were purchased from Cell Signaling Technology (Beverly, MA, USA). Anti- $\alpha$-smooth muscle actin ( $\alpha$-SMA) (A5228) and antifibronectin (F3648) were obtained from Sigma (Sigma-Aldrich, St. Louis, MO, USA). Anti-PCNA (BM0104) and anti-collagen I (BA0325) were obtained from BOSTER (Wuhan, China). Antineutrophil gelatinase-associated lipocalin (NGAL) (sc515876) and anti-p53 (10442-1-AP) were, respectively, purchased from Santa Cruz Biotechnology (Dallas, TX, USA) and Proteintech (Rosemont, IL, USA).

\section{Immunoprecipitation}

Kidney tissues were lysed in lysis buffer (10 mM HEPES, pH 7.9, $1 \mathrm{~mm}$ EDTA, $0.8 \%$ Triton X-100, $15 \mathrm{mM} \mathrm{KCl,} 2 \mathrm{mM} \mathrm{MgCl}_{2}$, and 1 $\mathrm{mM}$ dithiothreitol) containing 1:100 protease inhibitor cocktail
(Millipore), and then centrifuged at $4{ }^{\circ} \mathrm{C}$ at 3,000 $\mathrm{g}$ for $10 \mathrm{~min}$. The pellets were lysed in a high salt buffer (lysis buffer with 420 $\mathrm{mM} \mathrm{NaCl}$ and $25 \%$ glycerol) for $30 \mathrm{~min}$. Protein complexes were obtained by incubating precleared lysates with either specific antibodies or normal IgG overnight at $4^{\circ} \mathrm{C}$, respectively [24]. Protein $\mathrm{A} / \mathrm{G}$ agarose beads were added to the protein lysis for $4 \mathrm{~h}$. The precipitates were washed in IP wash buffer (Tris $20 \mathrm{~mm}, \mathrm{pH} 7.5, \mathrm{NaCl}$ $150 \mathrm{~mm}$, EDTA $1 \mathrm{~mm}$, and 1\% Triton-100) 3 times before eluted in $2 \times$ loading buffer (Sigma).

\section{Histological and Immunohistochemical Analyses}

Two-micrometer paraffin-embedded kidney sections were stained with H\&E and Masson-trichrome staining, 4- $\mu \mathrm{m}$ paraffinembedded kidney sections were stained with Sirius staining, according to the manufacturer's protocol. Ten to fifteen randomly chosen fields in the cortex were evaluated for each mouse in a blinded manner under the microscope $(\times 400)$ and an average staining positive area (\%) was calculated.

Immunohistochemical staining was performed on $4-\mu \mathrm{m}$ paraffin-embedded kidney sections as previously described [25]. After citric acid ( $\mathrm{pH}$ 6.0) repairing, the sections were incubated with the primary antibody against p-histone H3 (ser10) (ab14955, Abcam, Cambridge, UK) and then detected with En Vision/HRP Kit (Dako, Carpinteria, CA, USA).

$N A D^{+}$Measurement

$\mathrm{NAD}^{+}$from the kidney cortex was measured with an $\mathrm{NAD}^{+} /$ NADH Quantitation Kit (S0175; Beyo-time Biotechnology, CHN) according to the manufacturer's instructions.

\section{Flow Cytometry Analysis}

HK-2 cells were washed with cold PBS and then stained with the Cycle TESTTM PLUS DNA Reagent Kit (Becton Dickinson, Franklin Lakes, NJ, USA) [26]. Distribution of cell cycle was evaluated using BD FACSCanto II flow cytometry. Data were analyzed using ModFit LT3.3 (BD, Topsham, ME, USA) and represented as percent cell in G0/G1, S, and G2/M stages.

\section{Statistical Test}

Data are expressed as the mean \pm SD. Comparisons between 2 groups were analyzed using an unpaired Student's $t$ test. The comparisons between groups were made using one-way ANOVA followed by the Student-Newman-Keuls test (SPSS software, version 19.0; SPSS, Inc., Chicago, IL, USA). $p<0.05$ was considered as statistically significant.

\section{Results}

\section{NAM Ameliorates Renal Fibrosis in UUO Model}

To investigate whether NAM supplementation could attenuate renal fibrosis, $\mathrm{C} 57 \mathrm{BL} / 6 \mathrm{~J}$ male mice were injected with NAM $(0.25 \mathrm{mg} / \mathrm{g}) 3$ days before UUO operation. After 7 days, the mice were sacrificed, and the kidneys were collected for analysis. As shown in Figure 1a, the $\mathrm{NAD}^{+}$content in fibrotic renal tissue was approximately one-third of that in the control kidney. NAM supplemen- 


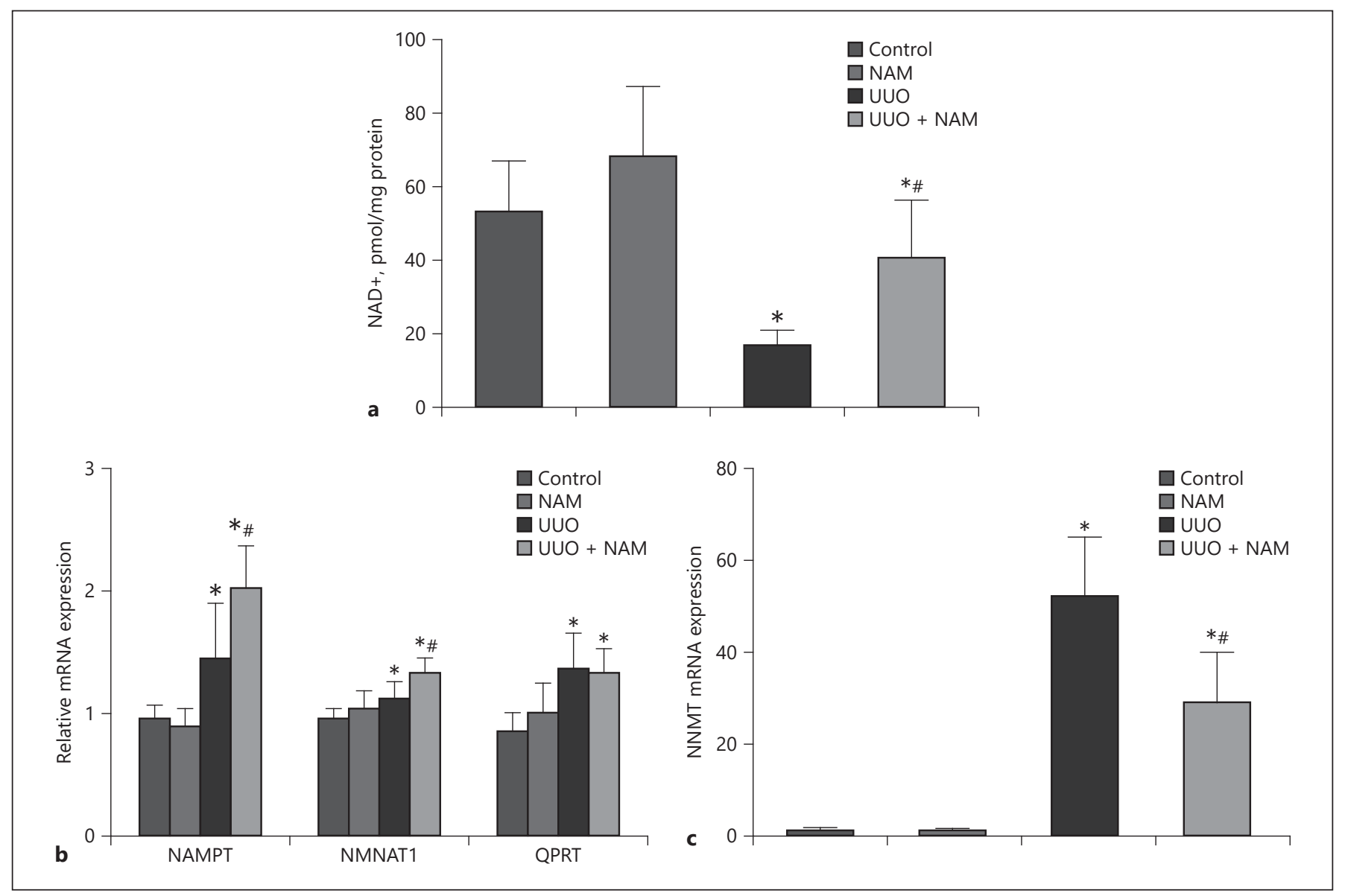

Fig. 1. Decreased $\mathrm{NAD}^{+}$level and disordered enzymes involved in NAM metabolism in UUO kidney. Mice received daily intraperitoneal injection of NAM $(0.25 \mathrm{mg} / \mathrm{g}) 3$ days before UUO, and were killed at day 7 after UUO operation. a The NAD ${ }^{+}$level was increased via injecting NAM ( $n=7$ each group). The relative mRNA expression of NAMPT, NMNAT1, QPRT (b) and NNMT in kidney cortexes of UUO7d model ( $n=6$ each group) (c). Data are expressed as mean \pm SD. ${ }^{*} p<0.05$ versus control group; ${ }^{*} p<0.05$ versus UUO group. NAM, nicotinamide; UUO, unilateral ureter obstruction; NAMPT, nicotinamide phosphoribosyltransferase; NMNAT1, nicotinamide mononucleotide adenylyltransferase1; QPRT, quinolinate phosphoribosyltransferase; NNMT, $N$-methyltransferase. tation significantly increased the $\mathrm{NAD}^{+}$content in the fibrotic kidney. Real-time PCR showed that the mRNA level of the rate-limiting salvage enzyme nicotinamide phosphoribosyltransferase (NAMPT) was significantly upregulated by NAM in fibrotic renal tissues. The expression of nicotinamide mononucleotide adenylyltransferase1 (NMNAT1), which promotes the conversion of NMN to NAD ${ }^{+}$, was slightly increased by NAM. In addition, the mRNA level of quinolinate phosphoribosyltransferase (QPRT), a bottleneck enzyme in the de novo biosynthesis of $\mathrm{NAD}^{+}$, was also upregulated in the fibrotic renal tissue. However, NAM supplementation did not modulate its expression (shown in Fig. 1b). Interestingly, the expression of NNMT, an enzyme which converts
NAM to MNAM, was significantly downregulated by NAM (shown in Fig. 1c). These data indicate that NAM supplementation promotes $\mathrm{NAD}^{+}$synthesis via salvage pathway. Meanwhile, NAM supplementation inhibits the metabolism of NAM to MNAM.

Masson and sirius staining showed that NAM reduced the deposition of fibers and collagen in fibrotic kidney (shown in Fig. 2a-d). Consistently, the protein levels of collagen I, fibronectin, tenascin $\mathrm{C}$, and $\alpha$-SMA were significantly decreased in the kidney tissue of NAM-treated mice (shown in Fig. 2e, f). In addition, the protein level of NGAL was significantly decreased, indicating that NAM attenuated tubular injury (shown in Fig. 2g, h). Real-time PCR revealed reduced expression of pro-inflammatory 


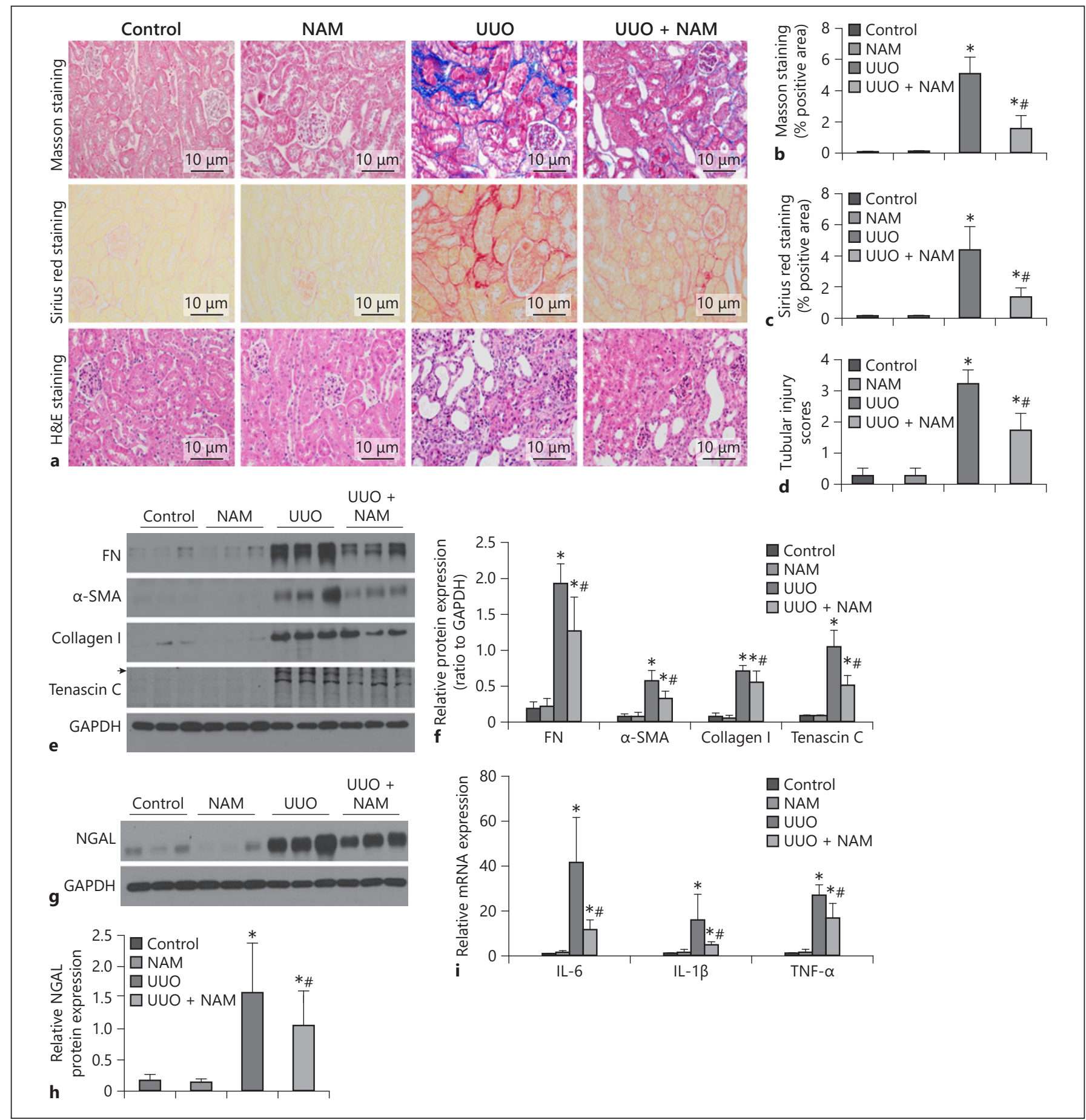

Fig. 2. NAM protects kidney from UUO-induced fibrosis. Mice received daily intraperitoneal injection of NAM $(0.25 \mathrm{mg} / \mathrm{g}) 3$ days before UUO and were killed at day 7 after UUO operation. a Representative micrographs show the renal interstitial fibrosis and tubular injury based on Masson staining, sirius red staining, and $\mathrm{H} \& \mathrm{E}$ staining and (b-d) were analyzed in a blind manner $(n=6$ each group). Scale bars, $100 \mu \mathrm{m}$. Western blots show the levels of FN, collagen I, tenascin C, $\alpha$-SMA, (e) and NGAL in kidney cortexes in different groups of the UUO7d model $(n=6)(\mathbf{g})$. f, $\mathbf{h}$ Graphic representation of relative protein levels was normalized against GAPDH. i Relative mRNA expression of IL-6, IL-1 $\beta$, and TNF- $\alpha$ in the kidney at different groups of UUO7d $(n=6$ each group). Data are expressed as mean $\pm \mathrm{SD} .{ }^{*} p<0.05$ versus control group; ${ }^{\#} p<0.05$ versus UUO group. NAM, nicotinamide; UUO, unilateral ureter obstruction; H\&E, hematoxylin-eosin; FN, fibronectin; $\alpha$-SMA, $\alpha$-smooth muscle actin; NGAL, neutrophil gelatinase-associated lipocalin; IL-6, interleukin-6; IL-1 $\beta$, interleukin-1 beta; TNF-a, tumor necrosis factor-alpha. 


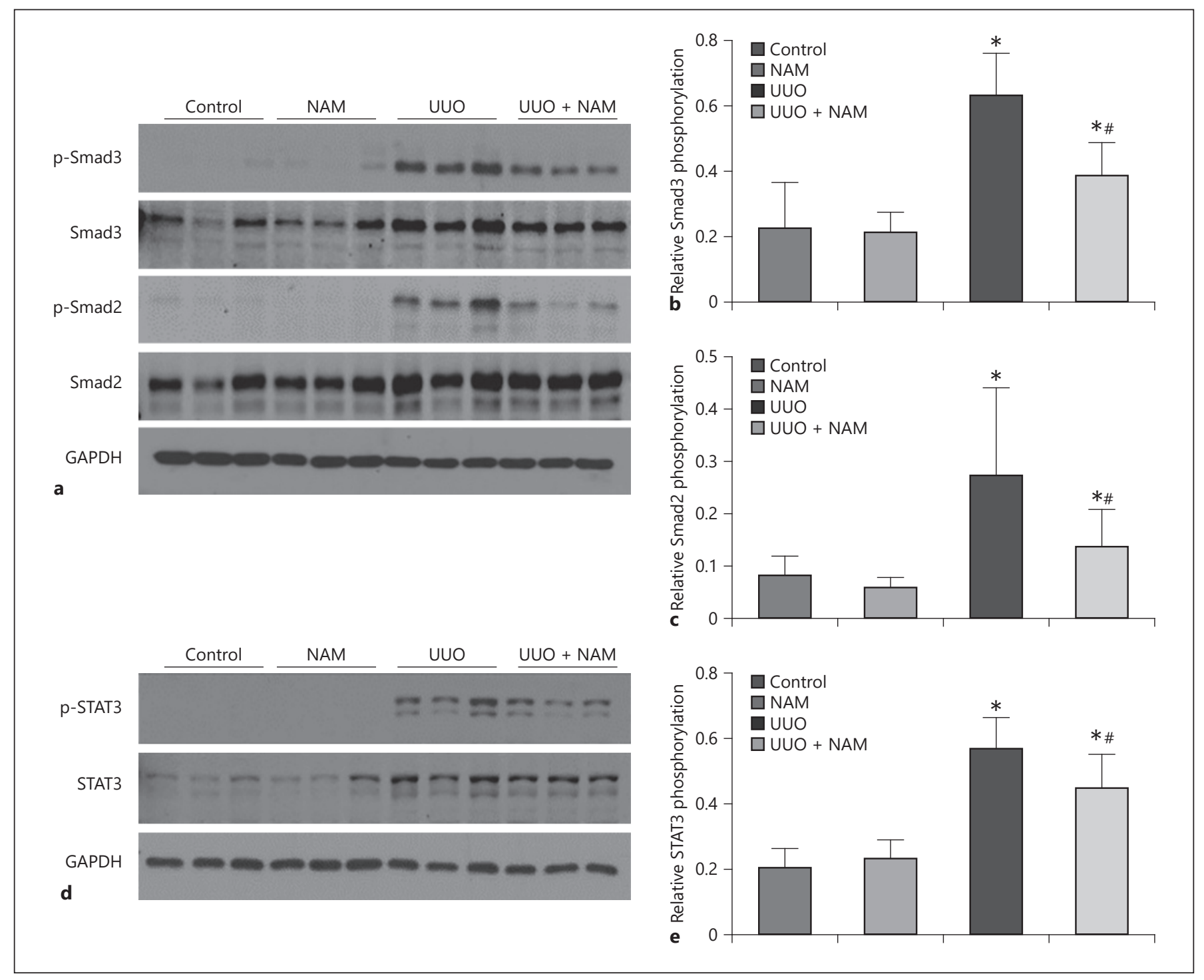

Fig. 3. NAM inhibits TGF- $\beta-S m a d 2 / 3$ and STAT3 signaling pathways in UUO model. Mice received daily intraperitoneal injection of NAM $(0.25 \mathrm{mg} / \mathrm{g}) 3$ days before UUO, and were killed at day 7 after UUO operation. a, d Representative Western blots show the levels of p-Smad3, p-Smad2, and p-STAT3 in kidney cortexes of different groups ( $n=6$ each group). b, c, e Graphic representation

cytokines including interleukin-1 $\beta$ (IL-1 $\beta$ ), interleukin-6 (IL-6), and tumor necrosis factor $\alpha$ (TNF- $\alpha$ ) in the kidney tissue of NAM-treated mice (shown in Fig. 2i). Mechanistically, NAM reduced the level of phosphorylated Smad3, Smad2, and STAT3 in fibrotic kidney tissues (shown in Fig. 3a-e), suggesting the reduced activation of Smad and STAT signaling pathways. Collectively, these data indicated that NAM attenuates interstitial fibrosis induced by UUO.

NAM Ameliorates Renal Fibrosis via Sirtuins of relative protein levels of $\mathrm{p}$-Smad3, p-Smad2, and p-STAT3 normalized against their total protein. Data are expressed as mean \pm SD. ${ }^{*} p<0.05$ versus control group; ${ }^{*} p<0.05$ versus UUO group. NAM, nicotinamide; UUO, unilateral ureter obstruction; $\mathrm{p}$ Smad3, phosphorylated Smad3; p-Smad2, phosphorylated Smad2; p-STAT3, phosphorylated STAT3.

\section{NAM Inhibited TGF- $\beta 1$-Induced Dedifferentiation of} Renal Tubular Cells

The effect of NAM on NGAL expression promoted us to investigate the role of NAM on tubular cells injury. We preincubated primary PTCs with NAM for $1 \mathrm{~h}$ before adding TGF- $\beta 1$. Consistent with in vivo data, NAM significantly inhibited TGF- $\beta 1$-induced expression of fibronectin, $\alpha$-SMA, collagen $I$, and tenascin $C$ (shown in Fig. $4 a, b)$. NAM treatment also inhibited TGF- $\beta 1$ - 
induced expression of IL-1 $\beta$, IL-6, TNF- $\alpha$, and MCP-1 (shown in Fig. 4c, d). Mechanistically, NAM attenuated TGF- $\beta 1$-induced phosphorylation of Smad3 (shown in Fig. 4e, f).

Since cell cycle arrest of PTCs at the G2/M phase contributes to the pathogenesis of interstitial fibrosis [7], we next investigated the effect of NAM on cell cycle. HK-2 cells were preincubated with NAM before adding AA. Flow cytometry analysis demonstrated that AA significantly increased the $\mathrm{G} 2 / \mathrm{M}$ population from 5.73 to $32.06 \%$ and NAM dramatically reduced the G2/M population after AA treatment from 32.06 to $10.03 \%$ (shown

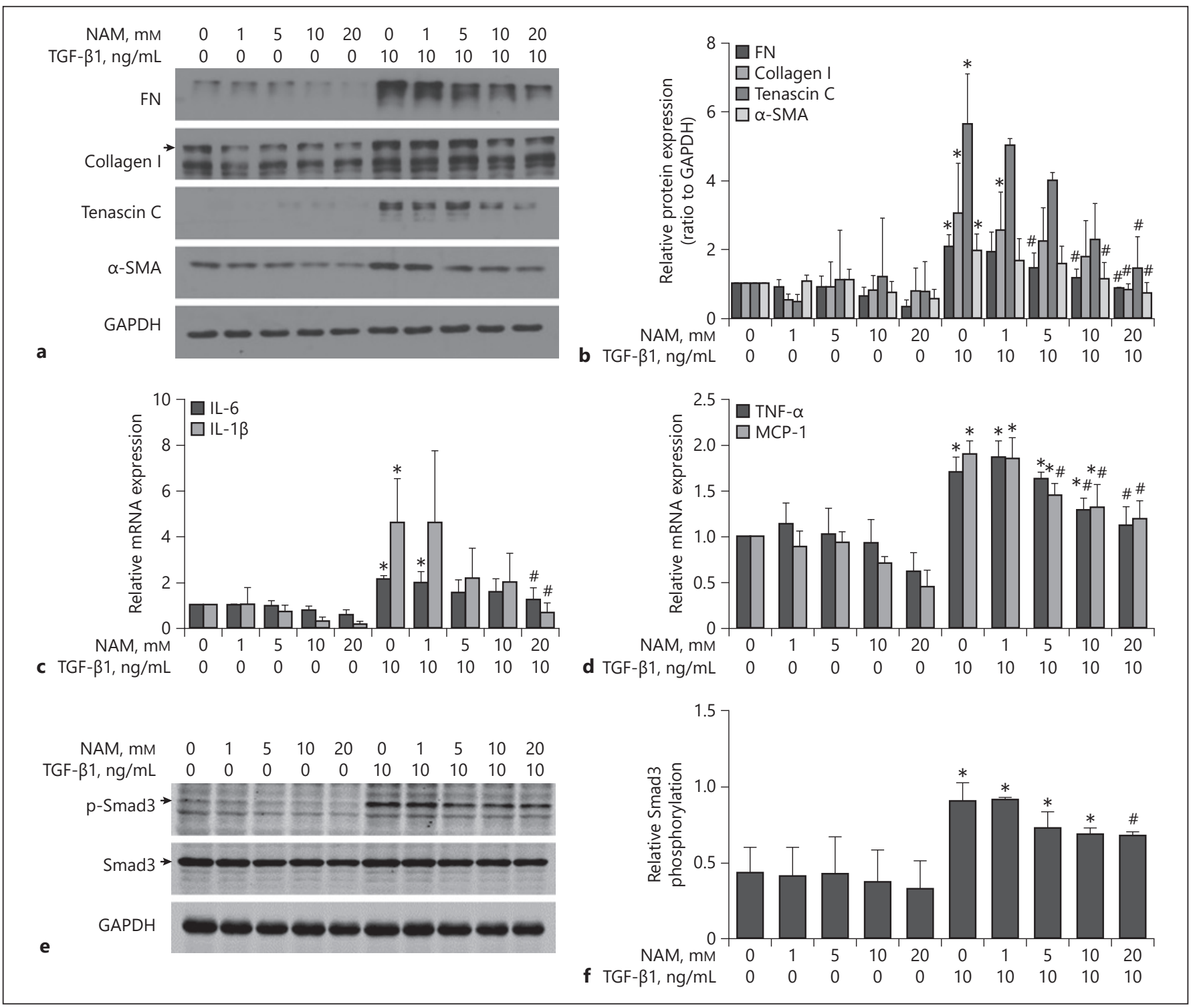

Fig. 4. NAM ameliorates the fibrosis caused by TGF- $\beta 1$ in primary PTCs. PTCs were pretreated with or without NAM $(1,5,10,20$ $\mathrm{mM}$ ) for $1 \mathrm{~h}$ and then co-incubated with TGF- $\beta 110 \mathrm{ng} / \mathrm{mL}$ for $24 \mathrm{~h}$. a Western blot analysis of FN, collagen I, tenascin C, and a-SMA. b Graphic representation of relative protein levels were normalized against GAPDH. c, d Relative mRNA expression of IL-6, IL-1 $\beta$, TNF- $\alpha$, and MCP-1. e Representative Western blotting images of phosphorylated and total Smad3.f Graph represents relative level of p-Smad3 normalized with Smad3. The data are representative of 3 independent experiments. Data are expressed as mean \pm SD. ${ }^{*} p<0.05$ versus the control group; ${ }^{\#} p<0.05$ versus TGF- $\beta 1$ (10 ng/mL) group. NAM, nicotinamide; PTCs, proximal tubular epithelial cells; TGF- $\beta 1$, transforming growth factor- $\beta 1$; FN, fibronectin; $\alpha$-SMA, $\alpha$-smooth muscle actin; IL- 6 , interleukin-6; IL- $1 \beta$, interleukin- 1 beta; TNF- $\alpha$, tumor necrosis factor-alpha; MCP-1, monocyte chemoattractant protein-1. 
in Fig. 5a, b). In the UUO model, cells in the G2/M phase were detected by staining of phosphorylated histone $\mathrm{H} 3$ at ser10 (p-H3). The number of $\mathrm{p}-\mathrm{H} 3$ positive tubular cells was significantly decreased in the kidney of NAMtreated mice (shown in Fig. 5c, d). In addition, we established a mouse model of renal damage induced by intra- peritoneal injection of AA $(3.5 \mathrm{mg} / \mathrm{kg})$. NAM was given 1 day before AA injection. Fifteen days after AA injection, mice were sacrificed and kidney tissues were collected for analysis. H\&E staining showed that NAM attenuated AA-induced renal tubular injury (shown in Fig. 5e, f). Consistently, the protein level of NGAL, a marker of in-

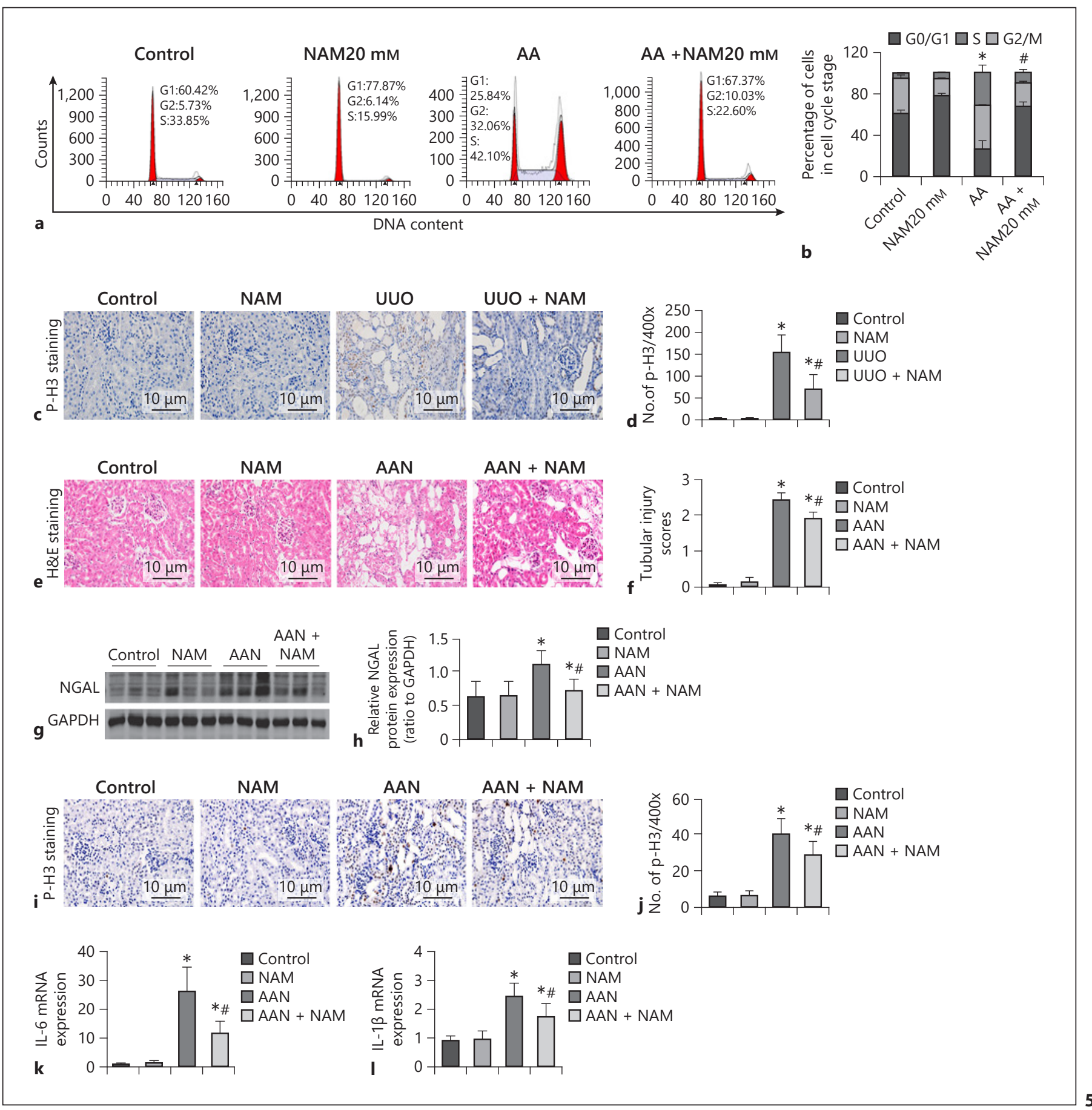

(For legend see next page.)

NAM Ameliorates Renal Fibrosis via Sirtuins
Kidney Dis 2021;7:186-199 DOI: $10.1159 / 000510943$ 
jured tubules, was decreased by NAM treatment (shown in Fig. 5g, h). Immunohistochemistry staining showed that the number of $\mathrm{p}-\mathrm{H} 3$-positive cells was reduced by NAM (shown in Fig. 5i, j). In addition, the mRNA levels of IL- 6 and IL- $1 \beta$ were also decreased (shown in Fig. 5k, 1). These data indicated that NAM attenuated renal tubular injury.

\section{NAM Inhibited TGF- $\beta 1$-Induced Fibroblasts \\ Proliferation and Activation}

Given the critical role of fibroblast proliferation and activation in interstitial fibrosis, we preincubated NRK$49 \mathrm{~F}$ cells with NAM for $1 \mathrm{~h}$ before adding TGF- $\beta 1$. NAM significantly inhibited TGF- $\beta 1$-induced expression of PCNA, cyclin D1, fibronectin, $\alpha$-SMA, collagen I, and tenascin $\mathrm{C}$ (shown in Fig. 6a-e). NAM treatment also inhibited TGF- $\beta 1$-induced expression of IL- 6 and MCP-1 (shown in Fig. 6f). In addition, NAM attenuated TGF$\beta 1$-induced phosphorylation of both Smad3 and STAT3 in a dose-dependent manner (shown in Fig. $6 \mathrm{~g}-\mathrm{i}$ ). These data suggest that NAM attenuated TGF- $\beta 1$-induced fibroblast activation via inhibiting both Smad and STAT3 signaling.

\section{SIRT1 Mediated the Beneficial Effect of NAM on TIF}

Previous studies showed that NAD modulates the activity of SIRT1, which deacytelates various signaling protein including Smad3 and p53 [27, 28]. To address this issue, we examined the level of acetylated FoxO1 [29]. As expected, immunoprecipitation assay revealed that the increased acetylation of FoxO1 in fibrotic kidney tissues was inhibited by NAM treatment (shown in Fig. 7a, b). As expected, the renal level of acetylated $\operatorname{Smad} 3$ was dramatically attenuated by NAM administration (shown in

Fig. 5. NAM ameliorates the tubular injury via inhibiting G2/M arrest. HK-2 cells were pretreated with NAM (20 mM) $1 \mathrm{~h}$ before being co-incubated with AA $15 \mu \mathrm{g} / \mathrm{mL}$ for $48 \mathrm{~h}$. a Cell cycle analysis by propidium iodide staining and flow cytometry in HK-2 cells in different groups. b Cell cycle distribution in HK-2 cells with or without previous NAM treatment. The data are representative of 3 independent experiments. Representative micrographs of immunohistochemical staining of $\mathrm{p}-\mathrm{H} 3$ in kidney of NAM-treated UUO model (c), and quantitative assessment of positive number of $\mathrm{p}-\mathrm{H} 3$ are presented. Mice were subject to intraperitoneal injection of AA every other day for 10 days (d). NAM $(0.5 \mathrm{mg} / \mathrm{g} /$ day $)$ was given 1 day before injection with AA till day 15 after initial injection of AA, and the mice were sacrificed. Representative micrographs of H\&E staining in kidney of NAM-treated AAN model (e), and quantitative assessment of tubular injury scores (f). g Western blots show the levels of NGAL in kidney cortexes in dif-
Fig. 7c, d). Similarly, the increased acetylation of p53 in fibrotic kidney tissues was also inhibited by NAM administration (shown in Fig. 7e, f). As expected, the upregulated mRNA level of cyclin G1 (CG1), a target gene of p53, in fibrotic kidney was inhibited by NAM administration (shown in Fig. 7g).

To further confirm that SIRT1 mediates the protective effect of NAM, primary PTCs were pretreated with EX527, a SIRT1 inhibitor, before adding NAM and TGF- $\beta 1$. Western blotting showed that NAM blocked TGF- $\beta 1$-augmented expression of fibronectin, collagen I, and $\alpha$-SMA, whereas EX527 pretreatment abolished the protective effect of NAM (shown in Fig. 8a, b). In addition, we pretreated primary PTCs with either resveratrol, an activator of SIRT1, or NAM, or NAM together with resveratrol before TGF- $\beta 1$ treatment. As shown in Figures $8 \mathrm{c}$ and $\mathrm{d}$, either NAM or resveratrol significantly inhibited TGF- $\beta 1$-induced expression of fibronectin, collagen I, and $\alpha$-SMA. Nevertheless, supplementation of resveratrol on the basis of NAM did not further decrease their expressions. Collectively, these in vivo and in vitro data suggest that NAM attenuated renal fibrosis at least partially via boosting the activity of SIRT1.

\section{Discussion}

It has been reported that NAM supplementation could prevent and reverse established AKI in a PGC-1 $\alpha$ dependent manner [19]. In this study, we demonstrate that NAM supplementation attenuated TIF induced by UUO and AA-induced renal tubular injury. NAM significantly inhibited G2/M arrest of PTCs shown as increased p-H3-positive cells and upregulation of CG1. In

ferent groups of the AAN model. $\mathbf{h}$ Graphic representation of relative protein levels were normalized against GAPDH. Representative micrographs of immunohistochemical staining of $\mathrm{p}-\mathrm{H} 3$ in kidney of NAM treated AAN model (i) and quantitative assessment of positive number of $\mathrm{p}-\mathrm{H} 3(\mathbf{j})$. Relative mRNA expression of IL-6 (k) and IL-1 $\beta$ (I) in the kidney at different groups of the AAN model. All tissue staining data are representative of at least 10 scopes at high power field independent experiments. Scale bars, $100 \mu \mathrm{m} . n=6$ each group. Data are expressed as mean \pm SD. ${ }^{*} p<$ 0.05 versus control group; ${ }^{\#} p<0.05$ versus AAN or UUO group. NAM, nicotinamide; AA, aristolochic acid; HK-2, human kidney proximal tubular epithelial cells; $\mathrm{p}-\mathrm{H} 3$, phosphorylated histone $\mathrm{H} 3$ at ser10; UUO, unilateral ureter obstruction; AAN, aristolochic acid toxic nephropathy; NGAL, neutrophil gelatinase-associated lipocalin; IL-6, interleukin-6; IL-1 $\beta$, interleukin-1 beta 
$\begin{array}{lllllllllll}\mathrm{NAM}, \mathrm{mM} & 0 & 1 & 5 & 10 & 20 & 0 & 1 & 5 & 10 & 20\end{array}$

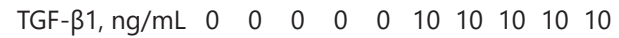

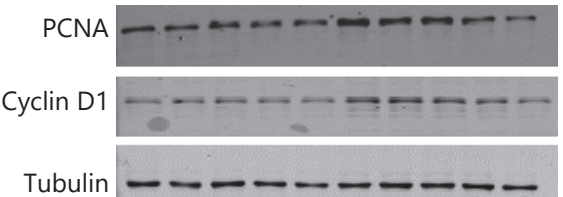

$\begin{array}{lllllllllll}\mathrm{NAM}, \mathrm{mM} & 0 & 1 & 5 & 10 & 20 & 0 & 1 & 5 & 10 & 20\end{array}$

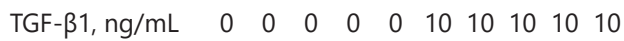

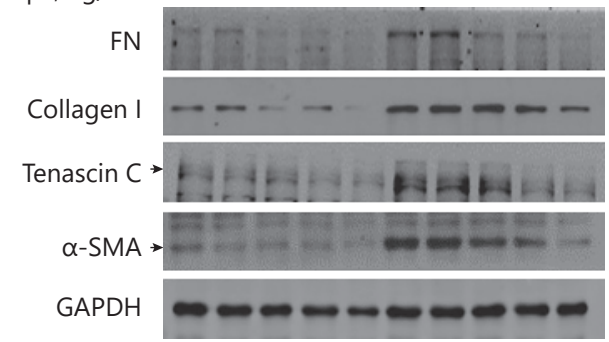

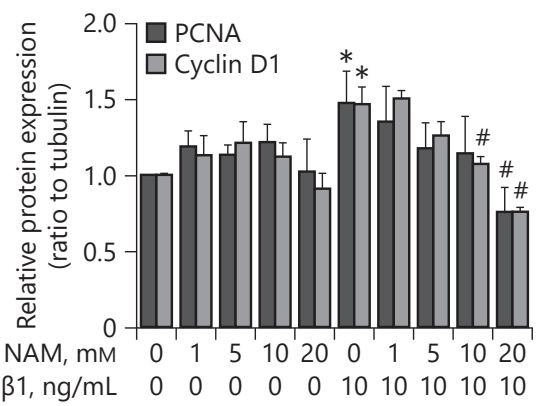

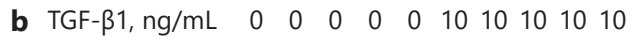

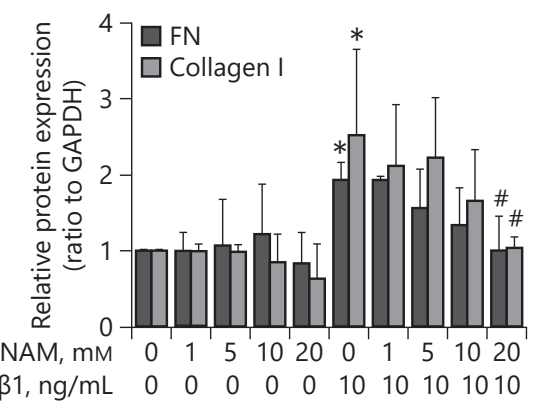

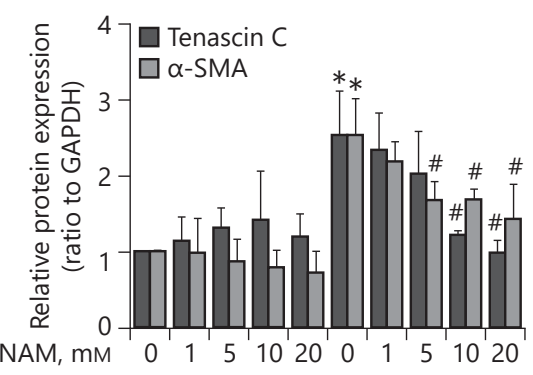

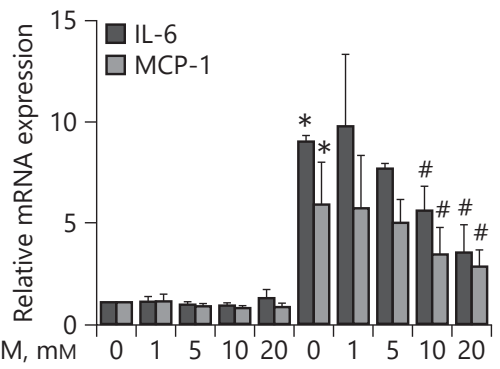

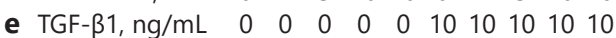
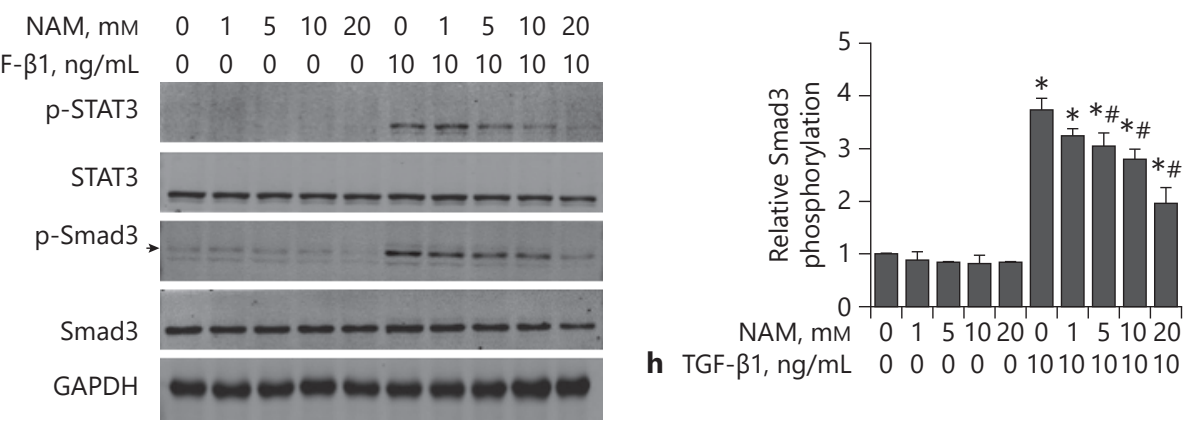

h $\mathrm{TGF}-\beta 1, \mathrm{ng} / \mathrm{mL} \quad 0 \quad 0 \quad 000001010101010 \quad$ i

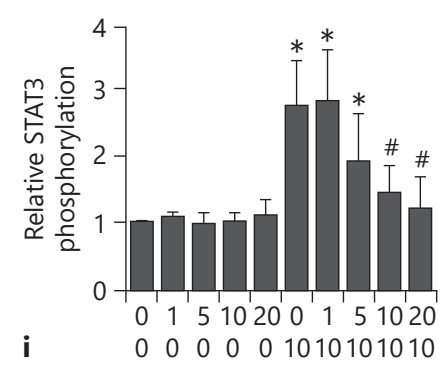

Fig. 6. NAM ameliorates the proliferation and activation in NRK$49 \mathrm{~F}$ caused by TGF- $\beta 1$. NRK- $49 \mathrm{~F}$ cells were preincubated with $\operatorname{NAM}(1,5,10,20 \mathrm{~mm}) 1 \mathrm{~h}$ before TGF- $\beta 110 \mathrm{ng} / \mathrm{mL}$ for $24 \mathrm{~h}$. Western blots show the expression of PCNA, cyclin D1 (a) and FN, collagen I, tenascin $\mathrm{C}$, and $\boldsymbol{\alpha}$-SMA (c). b, d, e Graphic representation of relative protein levels were normalized against tubulin or GAP$D H$. $\mathbf{f}$ Relative mRNA expression of IL- 6 and IL- $1 \beta$. Western blotting analysis of phosphorylation and total Smad3 and STAT3 are shown $(\mathbf{g})$, the relative protein expressions are normalized against total Smad3 and STAT3 $(\mathbf{h}, \mathbf{i})$. The data are representative of 3 independent experiments. Data are expressed as mean $\pm \mathrm{SD} .{ }^{*} p<$ 0.05 versus the control group; ${ }^{*} p<0.05$ versus TGF- $\beta 1(10 \mathrm{ng} / \mathrm{mL})$ group. NAM, nicotinamide; TGF- $\beta 1$, transforming growth factor- $\beta 1$; FN, fibronectin; $\alpha$-SMA, $\alpha$-smooth muscle actin; IL-6, interleukin-6; IL-1 $\beta$, interleukin-1 beta. 
Fig. 7. NAM ameliorates renal fibrosis via decreasing acetylated levels of Smad3 and p53. Western blotting analysis of acetylated and total protein of FoxO1, Smad3, and p53 in kidney cortexes (a, c, e), relative protein expression of acetylation were normalized against total protein $(n=5-6$ each group) (b, d, f). g Relative mRNA expression of cyclin G1 in kidney cortexes in different groups of UUO7d $(n=8)$. Data are expressed as mean \pm SD. ${ }^{*} p<0.05$ versus the control group; ${ }^{*} p<0.05$ versus UUO group. NAM, nicotinamide; FoxO1, forkhead box O1; UUO, unilateral ureter obstruction.

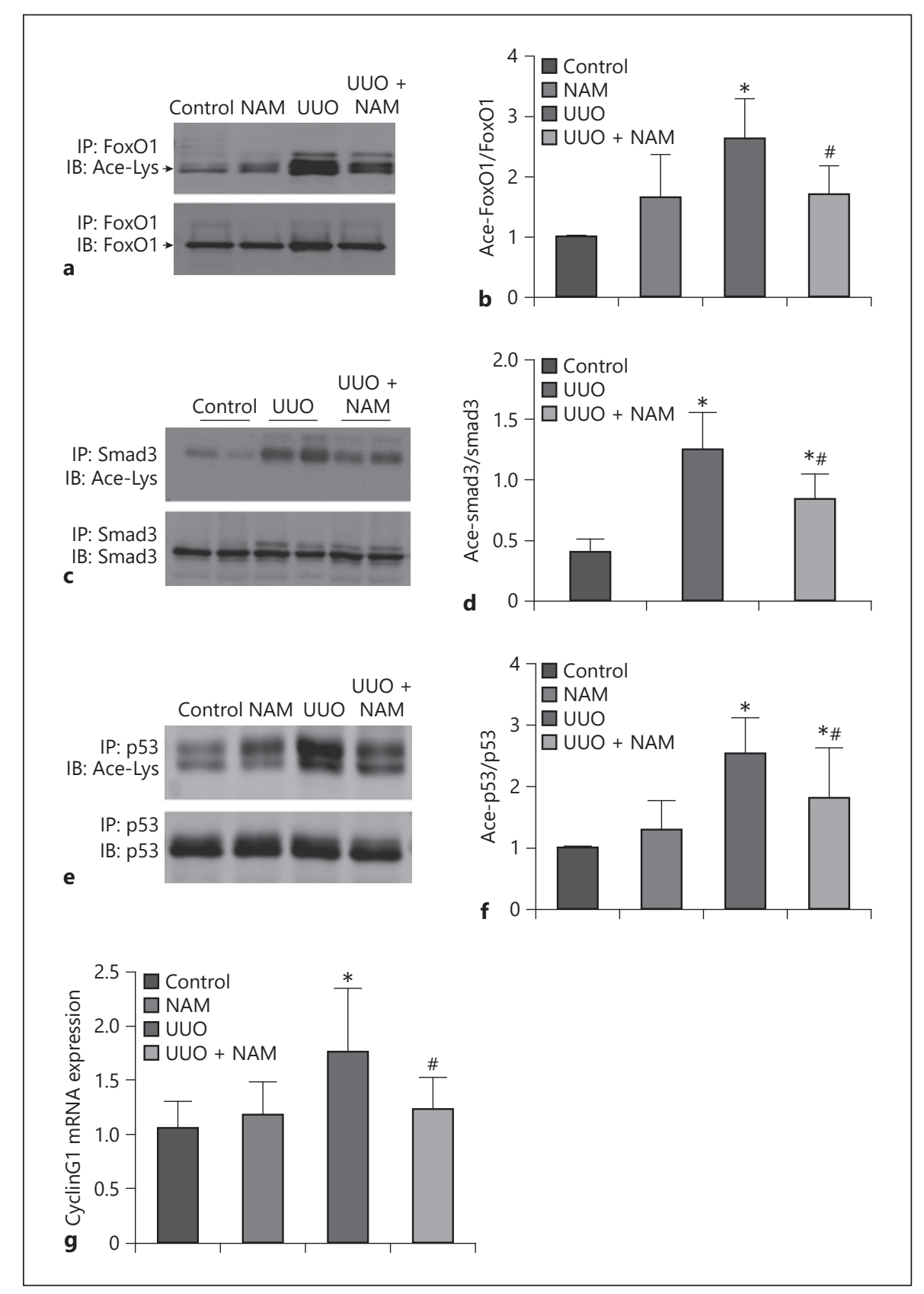

addition, NAM inhibited TGF- $\beta 1$-induced activation of fibroblast, which was indicated by reduced expression of PCNA, cyclin D1, fibronectin, collagen I, a-SMA, and pro-inflammatory cytokines. These data indicate that NAM supplementation exerts an anti-fibrotic effect via attenuating both proximal tubular cells injury and fibroblasts activation.

Previous studies showed that renal tubular cell cycle arrest in the G2/M phase of the cell cycle contributes to the pathogenesis of TIF by secretion of pro-fibrotic fac- tors and pro-inflammatory cytokines. In the current study, we demonstrated that NAM attenuated G2/M arrest of PTCs. Mechanistically, we showed that NAM inhibited the acetylation of p53 and subsequently downregulated the expression of CG1. Fibroblasts activation is another important factor driving the progression of TIF. We showed that NAM inhibited TGF- $\beta 1$-induced fibroblasts proliferation and activation. In addition, we found that NAM significantly inhibited the acetylation of Smad3. Since SIRT1 modulates the acetylation of p53 and 


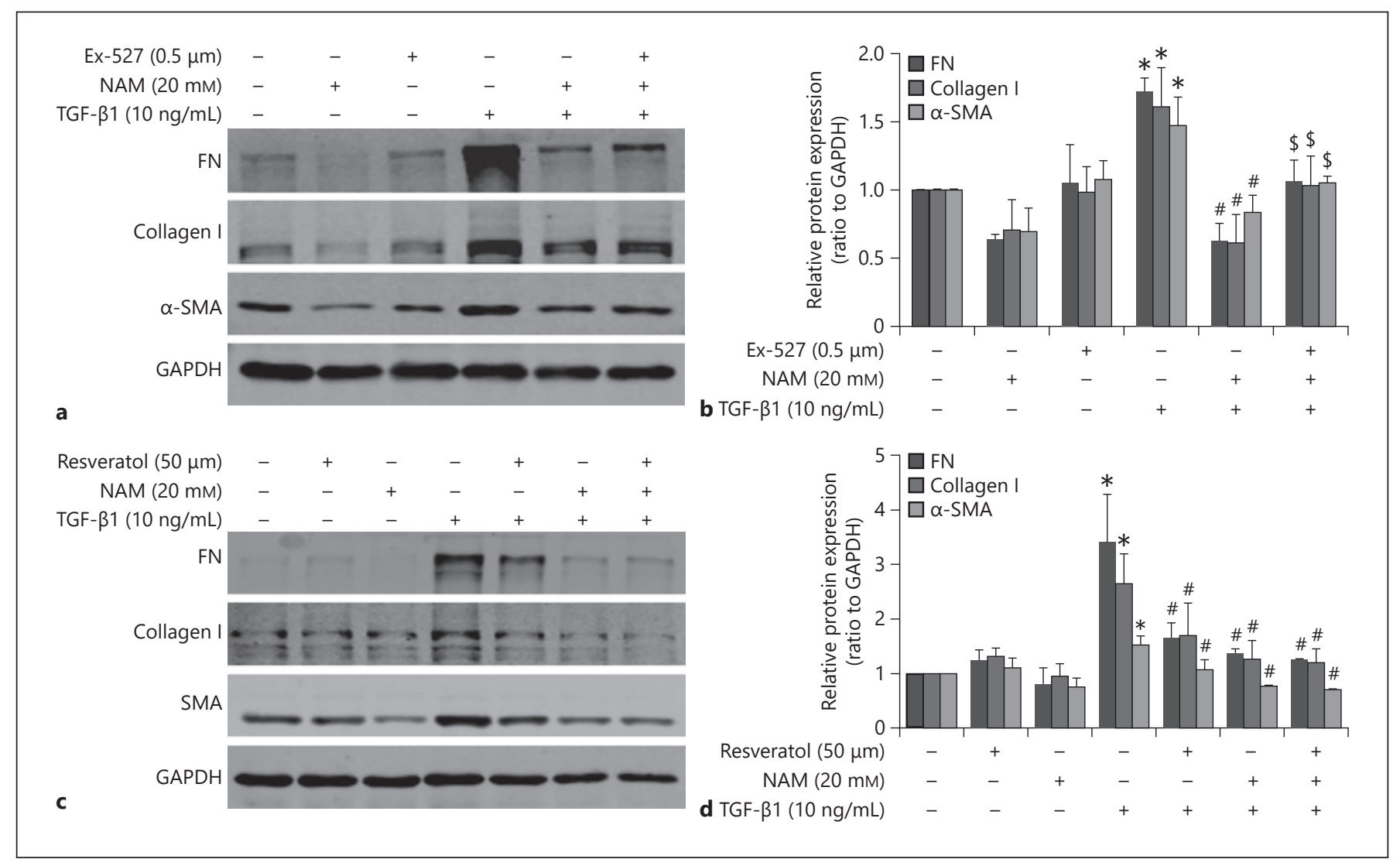

Fig. 8. NAM reduces TGF- $\beta 1$-induced matrix protein production through SIRT1. PTCs were preincubated with EX527 $(0.5 \mu \mathrm{M})$ or resveratrol $(50 \mathrm{mM})$ for $30 \mathrm{~min}$ and then treated with NAM (20 $\mathrm{mM}$ ) for $30 \mathrm{~min}$. Thereafter, PTCs were incubated with $10 \mathrm{ng} / \mathrm{mL}$ TGF- $\beta 1$ for 24 h. a, c Western blot analysis of FN, collagen I, and a-SMA. b, d Graphic representation of relative protein levels was normalized against GAPDH. The data are representative of 3 in- dependent experiments. Data are expressed as mean $\pm \mathrm{SD} .{ }^{*} p<$ 0.05 versus the control group; ${ }^{*} p<0.05$ versus TGF- $\beta 1(10 \mathrm{ng} / \mathrm{mL})$ group; ${ }^{\$} p<0.05$ versus the TGF- $\beta 1+$ NAM group. NAM, nicotinamide; TGF- $\beta 1$, transforming growth factor- $\beta 1$; SIRT1, sirtuins; PTCs, proximal tubular epithelial cells; FN, fibronectin; $\alpha$-SMA, a-smooth muscle actin.
Smad3 [27], we hypothesized that, in fibrotic renal tissues, $\mathrm{NAD}^{+}$depletion inhibits the activity of SIRTs, and NAM supplementation enhances the activity of SIRTs via replenishing $\mathrm{NAD}^{+}$contents. This hypothesis was supported by the following findings: first, NAM supplementation decreased the level of acetylated FoxO1 in fibrotic tissues. Second, NAM supplementation decreased the level of acetylated p53 and Smad3. Third, EX527, an inhibitor of SIRT1, abolished the inhibitory effect of NAM on TGF- $\beta 1$-induced ECM protein production in PTCs. These findings are supported by a previous report that NMN supplementation attenuates AKI injury in aging mice dependent on SIRT1 [30].

A previous study reported that, in the kidney of aging mice, the reduced $\mathrm{NAD}^{+}$contents were accompanied with a deficit in the salvage pathway [31]. However, in the current study, we found that the mRNA levels of NAMPT and NMNAT, which are rate-limiting enzymes in the $\mathrm{NAD}^{+}$salvage pathway, were significantly elevated in fibrotic renal tissues. Moreover, it has been reported that the QPRT, a bottleneck enzyme in the de novo biosynthesis of $\mathrm{NAD}^{+}$, is enriched in the kidney, and the mRNA level of QPRT was downregulated during ischemia-reperfusion induced AKI [12]. However, we detected an upregulation of QPRT mRNA in fibrotic renal tissue. These data suggest that the reduction of $\mathrm{NAD}^{+}$content in fibrotic renal tissue was not caused by a deficit in biosynthesis. $\mathrm{NAD}^{+}$is a co-substrate for $\mathrm{NAD}^{+}$-consuming enzymes. In addition to SIRTs, PARP-1 is another NAD ${ }^{+}$consuming enzyme. PARP-1 transfers ADP ribose from $\mathrm{NAD}^{+}$to itself, histones, and other proteins at sites of DNA damage to facilitate repair. There was a systemic 
elevation in $\mathrm{NAD}^{+}$levels and SIRT1 activity in PARP-1 knockout mice. Therefore, the activation of PARP-1 has been considered as a mechanism of $\mathrm{NAD}^{+}$decline [30]. Since DNA damage has occurred in injured tubular cells, it is possible that overactive PARP- 1 triggers $\mathrm{NAD}^{+}$depletion and contributes to the reduced activity of sirtuins in the fibrotic renal tissue.

$\mathrm{NAD}^{+}$depletion, as a key feature of renal disorders, plays a crucial role in the pathogenesis of AKI, CKD, and kidney aging [31]. It has been shown that systemic supplementation of NAM reverses IR-induced AKI. In addition, given the reported cardioprotective benefits of $\mathrm{NAD}^{+}$repletion [32], $\mathrm{NAD}^{+}$repletion might also benefit CKD patients with cardiovascular complications. In the current study, we provide evidence that NAM supplementation could attenuate TIF, supporting a beneficial effect of $\mathrm{NAD}^{+}$repletion on halting the progression of CKD. As such, stimulation of $\mathrm{NAD}^{+}$biosynthesis or supplementation with $\mathrm{NAD}^{+}$precursors might be potential therapeutic approaches to treat various kidney diseases. In addition to NAM, nicotinic acid and nicotinamide riboside are salvageable precursors of $\mathrm{NAD}^{+}$and hold the ability to increase $\mathrm{NAD}^{+}$ content [33]. Additional research is necessary to examine the differential effects of various $\mathrm{NAD}^{+}$precursors on renal diseases.

\section{Statement of Ethics}

All animal experiments conform to internationally accepted standards and have been approved by the Ethics Committee for Animal Experiments of the Southern Medical University.

\section{Conflict of Interest Statement}

The authors have no conflicts of interest to disclose.

\section{Funding Sources}

This work was supported by grants from Nature and Science Foundation of China $(81730019,81521003)$ and President Foundation of Nanfang Hospital, Southern Medical University (2017Z011) to Dr. Jing Nie and from President Foundation of Nanfang Hospital, Southern Medical University (2017Z019) to Dr. Fengxin Zhu.

\section{Author Contributions}

All authors participated in the study. Xin Zhen and Jing Nie contributed to the conception of the study and writing the manuscript. Xin Zhen, Shaowu Zhang, Feifei Xie, and Miaomiao Zhou conducted the experiments. Jing Nie supervised the work. Zheng $\mathrm{Hu}$ and Fengxin Zhu helped to run flow cytometry and data analyses. All authors have read and approved the final manuscript.

\section{References}

1 Gewin L, Zent R, Pozzi A. Progression of chronic kidney disease: too much cellular talk causes damage. Kidney Int. 2017 Mar;91(3): $552-60$.

2 Zhou D, Liu Y. Renal fibrosis in 2015: understanding the mechanisms of kidney fibrosis. Nat Rev Nephrol. 2016 Feb;12(2):68-70.

3 Breyer MD, Susztak K. The next generation of therapeutics for chronic kidney disease. Nat Rev Drug Discov. 2016 May;15(8):568-88.

4 Humphreys BD. Mechanisms of renal fibrosis. Annu Rev Physiol. 2018 Feb;80(6):30926.

5 Wu CF, Chiang WC, Lai CF, Chang FC, Chen YT, Chou YH, et al. Transforming growth factor $\beta-1$ stimulates profibrotic epithelial signaling to activate pericyte-myofibroblast transition in obstructive kidney fibrosis. Am J Pathol. 2013 Sep;182(1):118-31.

6 Ferenbach DA, Bonventre JV. Mechanisms of maladaptive repair after AKI leading to accelerated kidney ageing and CKD. Nat Rev Nephrol. 2015 May;11(5):264-76.

7 Yang L, Besschetnova TY, Brooks CR, Shah JV, Bonventre JV. Epithelial cell cycle arrest in G2/M mediates kidney fibrosis after injury. Nat Med. 2010 May;16(5):535-43.
8 Canaud G, Brooks CR, Kishi S, Taguchi K, Nishimura K, Magassa S, et al. Cyclin G1 and TASCC regulate kidney epithelial cell G2-M arrest and fibrotic maladaptive repair. Sci Transl Med. 2019 Jan;11(476):eaav4754.

9 Knip M, Douek IF, Moore WP, Gillmor HA, McLean AE, Bingley PJ, et al. Safety of highdose nicotinamide: a review. Diabetologia. 2000 Nov;43(11):1337-45.

10 Hershberger KA, Martin AS, Hirschey MD. Role of NAD+ and mitochondrial sirtuins in cardiac and renal diseases. Nat Rev Nephrol. 2017 Apr;13(4):213-25.

11 Frederick DW, Loro E, Liu L, Davila A, Chellappa K, Silverman IM, et al. Loss of NAD homeostasis leads to progressive and reversible degeneration of skeletal muscle. Cell Metab. 2016 Aug;24(2):269-82.

12 Poyan Mehr A, Tran MT, Ralto KM, Leaf DE, Washco V, Messmer J, et al. De novo NAD+ biosynthetic impairment in acute kidney injury in humans. Nat Med. 2018 Sept;24(9): 1351-9.

13 Pissios P. Nicotinamide N-methyltransferase: more than a vitamin b3 clearance enzyme. Trends Endocrinol Metab. 2017 May;28(5): $340-53$.
14 Wilhelm F, Hirrlinger J. The NAD+/NADH redox state in astrocytes: independent control of the NAD+ and NADH content. J Neurosci Res. 2011 Apr;89(12):1956-64.

15 Gardell SJ, Hopf M, Khan A, Dispagna M, Hampton Sessions E, Falter R, et al. Boosting $\mathrm{NAD}+$ with a small molecule that activates NAMPT. Nat Commun. 2019 Jul;10(1):3241.

16 Wakino S, Hasegawa K, Itoh H. Sirtuin and metabolic kidney disease. Kidney Int. 2015 Jun;88(4):691-8.

17 Zhang W, Huang Q, Zeng Z, Wu J, Zhang Y, Chen Z. Sirt1 inhibits oxidative stress in vascular endothelial cells. Oxid Med Cell Longev. 2017 May;2017:7543973-8.

18 Hwang ES, Song SB. Nicotinamide is an inhibitor of SIRT1 in vitro, but can be a stimulator in cells. Cell Mol Life Sci. 2017 Apr;74(18):3347-62.

19 Tran MT, Zsengeller ZK, Berg AH, Khankin EV, Bhasin MK, Kim W, et al. PGCla drives NAD biosynthesis linking oxidative metabolism to renal protection. Nature. 2016 Mar; 531(7595):528-32.

20 Wang B, Liu D, Zhu QH, Li M, Chen H, Guo $\mathrm{Y}$, et al. Rutin ameliorates kidney interstitial fibrosis in rats with obstructive nephropathy. Int Immunopharmacol. 2016 Jun;35:77-84. 
21 Liu Z, Li H, Su J, Xu S, Zhu F, Ai J, et al. Numb depletion promotes Drp1-mediated mitochondrial fission and exacerbates mitochondrial fragmentation and dysfunction in acute kidney injury. Antioxid Redox Signal. 2019 May;30(15):1797-816.

22 Fu X, He Y, Wang X, Peng D, Chen X, Li X, et al. Overexpression of miR-21 in stem cells improves ovarian structure and function in rats with chemotherapy-induced ovarian damage by targeting PDCD4 and PTEN to inhibit granulosa cell apoptosis. Stem Cell Res Ther. 2017 Aug;8(1):187.

23 Qi S, Song Y, Peng Y, Wang H, Long H, Yu X, et al. ZEB2 mediates multiple pathways regulating cell proliferation, migration, invasion, and apoptosis in glioma. Plos One. 2012 Jun; 7(6): 38842.

$24 \mathrm{Ni} \mathrm{W}$, Zhang Y, Zhan Z, Ye F, Liang Y, Huang J, et al. A novel lncRNA uc.134 represses hepatocellular carcinoma progression by inhibiting CUL4A-mediated ubiquitination of LATS1. J Hematol Oncol. 2017 Apr;10(1):91.
25 Song Y, Luo Q, Long H, Hu Z, Que T, Zhang $\mathrm{X}$, et al. Alpha-enolase as a potential cancer prognostic marker promotes cell growth, migration, and invasion in glioma. Mol Cancer. 2014 Mar; 13:65.

26 Sun Y, Zhou Y, Bai Y, Wang Q, Bao J, Luo Y, et al. A long non-coding RNA HOTTIP expression is associated with disease progression and predicts outcome in small cell lung cancer patients. Mol Cancer. 2017 Oct;16(1): 162.

27 Wang W, Sun W, Cheng Y, Xu Z, Cai L. Role of sirtuin-1 in diabetic nephropathy. J Mol Med. 2019 Mar;97(3):291-309.

28 Zhang J, Wang QZ, Zhao SH, Ji X, Qiu J, Wang J, et al. Astaxanthin attenuated pressure overload-induced cardiac dysfunction and myocardial fibrosis: partially by activating SIRT1. Biochim Biophys Acta. 2017 Jul; 1861(7):1715-28.

29 Hong S, Moreno-Navarrete JM, Wei X, Kikukawa Y, Tzameli I, Prasad D, et al. Nicotinamide $\mathrm{N}$-methyltransferase regulates hepatic nutrient metabolism through Sirt1 protein stabilization. Nat Med. 2015 Aug;21(8):887-94.

30 Imai S, Guarente L. NAD+ and sirtuins in aging and disease. Trends Cell Biol. 2014 Aug; 24(8):464-71.

31 Guan Y, Wang SR, Huang XZ, Xie QH, Xu YY, Shang D, et al. Nicotinamide mononucleotide, an NAD+ precursor, rescues age-associated susceptibility to AKI in a sirtuin 1-dependent manner. J Am Soc Nephrol. 2017 Aug;28(8):2337-52.

32 Matasic DS, Brenner C, London B. Emerging potential benefits of modulating NAD+ metabolism in cardiovascular disease. Am J Physiol Heart Circ Physiol. 2018 Apr;314(4): H839-52.

33 Braidy N, Berg J, Clement J, Khorshidi F, Poljak A, Jayasena T, et al. Role of nicotinamide adenine dinucleotide and related precursors as therapeutic targets for age-related degenerative diseases: rationale, biochemistry, pharmacokinetics, and outcomes. Antioxid Redox Signal. 2019 Jan;30(2):251-94. 